\title{
SN1987A: Temporal Models
}

\author{
M.I.Wanas, M.Melek and M.E.Kahil \\ Astronomy Department, Faculty of Science, Cairo University, Giza, \\ Egypt \\ E-mail: wanas@mailer.scu.eun.eg
}

\begin{abstract}
We construct two temporal models for SN1987A. The first model assumes spin independent trajectories for massless particles, while the second assumes spin dependent ones. The suggested scheme can be applied to any supernova event.
\end{abstract}

\section{Background}

Carriers of astrophysical information are massless spinning particles. These carriers are photons, neutrinos and expectedly gravitons. These three types of carriers are assumed to be emitted from supernovae events. In February, $23^{\text {rd }}, 1987$ a supernova of type II, in the Large Magellanic Cloud, was observed (cf.[1]). Observations of the arrival time of neutrinos, at the Kamiokande detectors, was recorded in February $23^{r d} 1987,7^{h} 35^{m} U T$, while the arrival time of photons was on the same day at $10^{h} 40^{m} U T$. The bar of the gravitational waves antennae in Rome and Maryland recorded relatively large pulses, 1.2 seconds earlier than neutrinos (cf.[2]). Although the three types of particles have different spins, general relativity assumes that they follow the same trajectory (null-geodesic of the metric), since all are massless. Recently, it is shown that spinning particles follow different trajectories [3]. It is shown that the spin dependent trajectory is given by,

$$
\frac{d Z^{\mu}}{d \tau}+\left\{\begin{array}{c}
\mu \\
\nu \sigma
\end{array}\right\} Z^{\nu} Z^{\sigma}=-\frac{n}{2} \alpha \gamma \Lambda_{(\nu \sigma)} \cdot{ }^{\mu} Z^{\nu} Z^{\sigma}
$$

where $Z^{\mu}$ is the tangent to the path, $\Lambda_{. \nu \sigma}^{\mu}$ is the torsion of space-time produced by the background gravitational field, $\alpha$ is the fine structure constant, $\gamma$ is a numerical parameter of order unity and $n$ is a natural number taking the value $0,1,2, \ldots$ for $0, \frac{1}{2}, 1, \ldots$-spin particles, respectively. Equation (1) reduces to null-geodesic upon taking $n=0$. Linearizing equation (1) using static weak gravitational field, we get a relation between the Newtonian potential $\Phi_{N}$ and the spin dependent potential $\Phi_{S}[3]$.

$$
\Phi_{S}=\left(1-\frac{n}{2} \alpha \gamma\right) \Phi_{N}
$$




\section{Temporal Models}

We mean by a temporal model, a model that can predict and arrange chronologically the emission times of different carriers, knowing their arrival times. In the context of general relativity, it is well known that the time interval required for a massless particle to traverse a given distance is longer in the presence of gravitational field having the potential $\Phi(r)$. The time delay is given by (cf.[4])

$$
\Delta t_{G R}=\text { const. } \int_{e}^{a} \Phi(r) d t
$$

where $e$ and $a$ are the emission and arrival times of the carrier, respectively. In the calculations of the time delay in the case of SN1987A (cf.[4]), $\Phi(r)$ is taken to be the Newtonian potential $\Phi_{N}$ (spin independent). In this case we get a spin independent model (SIM). If we assume that $\Phi(r)$ is the spin dependent gravitational potential $\Phi_{S}$, we then get the spin dependent model (SDM). The results of these two models are summarized in table I.

Table 1: Emission Times Given By The Models

\begin{tabular}{|c|c|c|}
\hline Particles Emitted & SIM & SDM \\
\hline Neutrino (core collapse) & 0.0 & 0.0 \\
\hline Photons (maximum brightness) & $+3^{h} 5^{m}$ & $+15^{h} 18^{m}$ \\
\hline Gravitons (?) & $-1^{s} .2$ & $+36^{h} 28^{m}$ \\
\hline
\end{tabular}

From the above table we can conclude that, the two models assume two different scenarios for the emission of carriers of astrophysical information. SIM shows an indication that neutrinos were emitted due to core collapse, associated with gravitons as a result of sudden change in the space-time symmetry due to a kicked born neutron star [5]. About three hours later photons were emitted as a result of maximum brightness of the envelope. SDM shows that: neutrinos were emitted due to a core collapse, preserving sphericity of the core. After 15 hours photons were emitted due to maximum brightness of the envelope, in agreement with SN theories (cf.[6]), then 21 hours later the envelope explodes asymetrically producing a sudden change of space-time symmetry and emits gravitational waves. It could be seen that SDM is more preferable than SIM.

\section{References}

1. Schramm, D. N. and Truran, J. W. 1990, Physics Reports, 189, 89

2. Weber, J. 1994, Proc. First Edoarndo Amaldi Conf. Ed. E.Coccia et al. World Scientific 416

3. Wanas, M. I. 1998, Astrophys. Space Sci., 258, 237

4. Krauss, L. M. and Tremaine, S. 1988, Phys. Rev. Lett., 60, 176

5. Kohri, K. and Nagantaki, S. 1999, astro-ph/9911077

6. De Rujula, A. 1988, Preprint CERN-TH. 5012/88 